\title{
Brain Representation of Active and Passive Hand Movements in Children
}

\author{
ANDREA GUZZETTA, MARTIN STAUDT, ELISA PETACCHI, JAN EHLERS, MICHAEL ERB, MARKO WILKE, \\ INGEBORG KRÄGELOH-MANN, AND GIOVANNI CIONI
}

\begin{abstract}
Department of Developmental Neuroscience [A.G., E.P., G.C.], Stella Maris Scientific Institute, I-56018 Calambrone, Pisa, Italy; Division of Child Neurology and Psychiatry [E.P., G.C.], University of Pisa, I-56018 Calambrone, Pisa, Italy; Department of Pediatric Neurology and Developmental Medicine [A.G., M.S., J.E., M.W., I.K.-M.], University Children's Hospital, D-72076 Tübingen, Germany; Department of Neuroradiology [M.S., J.E., M.E., M.W.], Radiologic Clinic, University of Tübingen, D-72076 Tübingen, Germany
\end{abstract}

\begin{abstract}
Several functional neuroimaging studies have been performed exploring the sensorimotor function in children with neurologic disorders. However, little is known about normal activation patterns of the sensorimotor system at a young age. We explored brain representation of active and passive hand movements in schoolage children and young adults. Nine healthy children (7-15 y) and six adults were studied. Functional magnetic resonance imaging (fMRI) data were acquired on a 1.5-T scanner in block designs. Active movement consisted of repetitive opening and closing of the hand; passive movement consisted of the same movement performed by the examiner. Both hands were assessed separately. The pattern of brain activation (contralateral primary sensorimotor cortex (SMC), ipsilateral cerebellum, supplementary motor area (SMA), and lateral premotor cortex (PMC) was generally more widespread in the adult group, suggesting a developmental course in the organization of both motor and sensory cortex. Surprisingly, no difference was generally detected when contrasting active versus passive tasks. Our results suggest that active and passive hand movements can be used for the exploration of the sensorimotor system in children. Passive and active tasks confirmed to be tightly coupled, thus supporting the idea of the former as a helpful performance-independent paradigm in the study of brain reorganization and presurgical assessment. (Pediatr Res 61: 485-490, 2007)
\end{abstract}

$\mathrm{B}^{\mathrm{r}}$ rain representation of sensorimotor function has been widely investigated in normal and pathologic subjects by means of functional neuroimaging techniques (1). For this purpose, active and passive hand or finger movements in sequences of various complexity are commonly used as activation tasks for the exploration of different components of the system.

Studies in adults have consistently shown significant activation of contralateral rolandic regions (i.e. the pre- and postcentral gyrus) both during active and passive movements, with different types of repetitive tasks. Other regions are also usually activated such as the SMA, the PMC, and the ipsilat-

Received July 25, 2006; accepted November 20, 2006.

Correspondence: Andrea Guzzetta, M.D., Department of Developmental Neuroscience, Stella Maris Scientific Institute, Via dei Giacinti, 2 I-56018 Calambrone (Pisa) Italy; e-mail: a.guzzetta@inpe.unipi.it

The study was supported by grant PRIN 2005 and FIRB 2001 of the Italian Minister of Scientific Research and University and by the Deutsche Forschungsgemeinschaft (SFB 550-C4).

DOI: $10.1203 /$ pdr.0b013e3180332c2e eral cerebellum (1-9). Surprisingly, when comparing active and passive tasks, very little differential activation has been generally shown, particularly at the level of the rolandic region, which could be expected to be differentially involved in the two conditions (9).

Little is known about brain representation of active and passive movements in children. Some knowledge about active motor tasks derives from studies with small control groups $(3,10)$, which showed similar patterns of activation to those reported in adult studies $(5,9,11,12)$. Nevertheless, the only study that systematically compared active movements in children and adults (repetitive hand squeezing of a ball) reported a significantly wider activation in the latter, particularly at the level of the primary SMC, the SMA, and the cerebellum (13). No studies on passive movement in normal children have been published so far, and no data are therefore available comparing children and adult passive movements or active and passive tasks in children.

In the present study, we investigated brain representation of active and passive hand movements in school-age children and in young adults. In particular, we wished to explore age-related differences in (i) brain representation of active movement, (ii) brain representation of passive movement, and (iii) differential activation between active and passive movements.

\section{SUBJECTS AND METHODS}

A total of nine healthy children (four girls; mean age $10.8 \mathrm{y}$, range 7-15 y) and six healthy young adults (three women; mean age $25 \mathrm{y}$, range 23 to 28 y) were studied. All subjects were right-handed and presented a normal neurologic status, normal neuroimaging (T1-weighted MRI), and an uneventful clinical history. Informed written consent from the subjects or their parents if they were underage was obtained. The study was approved by the Ethik Kommission der Medizinische Fakultät, Tübingen.

Imaging methods and activation tasks. All MRI measurements were performed on a conventional 1.5-T Siemens Avanto scanner (Siemens, Erlangen, Germany). Functional imaging data were acquired using a wholebrain multislice echo-planar imaging (EPI) sequence (14) [echo time (TE) = $50 \mathrm{~ms}, 1-\mathrm{mm}$ gap, 27 axial slices, voxel size $\left.3 \times 3 \times 4 \mathrm{~mm}^{3}\right]$ with an acquisition time (TA) of $2.4 \mathrm{~s}$ and an interscan interval (TR) of $6 \mathrm{~s}$ so that the scanning noise ceased for $3.6 \mathrm{~s}$ after each scan. The experiments were arranged in block designs, with alternations between four epochs of silent rest and four epochs of activation. Each epoch consisted of five scans, so that the

Abbreviations: BOLD, blood oxygen level dependent; fMRI, functional magnetic resonance imaging; IPL, inferior parietal lobule; PMC, premotor cortex; SMA, supplementary motor area; SMC, sensorimotor cortex 
total session comprised 40 scans. Two such sessions (active/passive) were performed for the left and the right hand in random order.

All subjects received detailed instructions before the measurement. During the active hand movement task, they were asked to repetitively open and close the hand at a frequency of $1 \mathrm{~Hz}$; the commands "now move" and "now pause" were given in the 3.6-s breaks between actual scanning at the beginning of the respective epochs; during the rest period, they were asked simply to lie still. The examiner controlled task performance visually. During the passive hand movement task, the subjects were asked to relax while the examiner repetitively opened and closed their hand at a frequency of $1 \mathrm{~Hz}$; during the rest period, the examiner kept holding the subject's hand and they were asked simply to lie still.

Image processing and data analysis. fMRI data postprocessing and statistical analysis of the functional images were performed using SPM2 (Statistical Parametric Mapping, Wellcome Department of Cognitive Neurology, University College, London, UK); default settings were used unless indicated otherwise. For a better exploration of our data, two types of analyses were performed: an individual analysis and a group analysis.

Individual analysis. In a first step of statistical analysis, after realignment, reslicing, and smoothing by means of an anisotropic Gaussian kernel of $6 \times$ $6 \times 8 \mathrm{~mm}^{3}$, we calculated activation maps for each subject individually. An activation threshold of $p<0.05$ was applied at the voxel level corrected for multiple comparisons family wise error (FWE) (15). Activation maps were calculated for individual assessments in each hand of (i) each task versus rest and (ii) active task versus passive task.

For a better comparison of the two groups to avoid biases due to different brain volumes between children and adults, stereotactic normalization was also performed, using the averaged functional EPI image and the SPM EPI template. The number of activated voxels in the SMC, the SMA, the lateral PMC, the inferior parietal lobule (IPL), and the cerebellum were then counted for each hemisphere in each subject. Comparisons of the activated volumes, expressed as the number of suprathreshold voxels, were performed with a Mann-Whitney $U$ test, using a $p<0.05$ threshold for statistical significance.

Group analysis. To obtain activation maps across subjects, the normalized functional data within both groups were combined in a fixed effect, which is a less conservative and more sensitive approach to identify activated brain areas after different tasks (16). A corrected activation threshold of $p<0.05$ was again applied at the voxel level (FWE).

Voxel-wise comparisons were performed as random-effect analyses of the two tasks (active versus passive and passive versus active in the two groups) and the two groups (children versus adults and adults versus children for the two tasks): after entering the contrast maps of the individual subjects' analyses into a second-level analysis, voxel-wise $t$ tests were performed to search for areas significantly more activated in one of the two tasks/groups. This random-effect analysis is considered the only valid approach to compare group data emerging from two different samples (16).

\section{RESULTS}

Brain representation of active movements in children and adults. In each comparison of active motor task and silent rest for both the children and the adult groups, a large cluster of significantly activated voxels was always found in the contralateral primary SMC. Activated areas were also present, in a variable proportion of subjects, in the cerebellum, the SMA, the PMC, and the IPL (Fig. 1a). The pattern of brain activation was generally more widespread in the adult group, as shown by the presence of a higher proportion of active regions in adults compared with children. This pattern was confirmed by the number of activated voxels that was significantly different in the two groups, particularly in the contralateral SMC ( $p<$ $0.05)$ and in the ipsilateral cerebellum $(p<0.01)$. No significant difference was found for the SMA (Fig. 2a).

A visual comparison of the fixed-effect activation patterns after active hand movement in the two groups revealed a high degree of similarity (Fig. 3). In both samples, the global maximum of activation was located in the contralateral rolandic region. Other large clusters of activation were detected in the ipsilateral cerebellum and the SMA, whereas a smaller one was observed in the contralateral IPL (corresponding to sec-

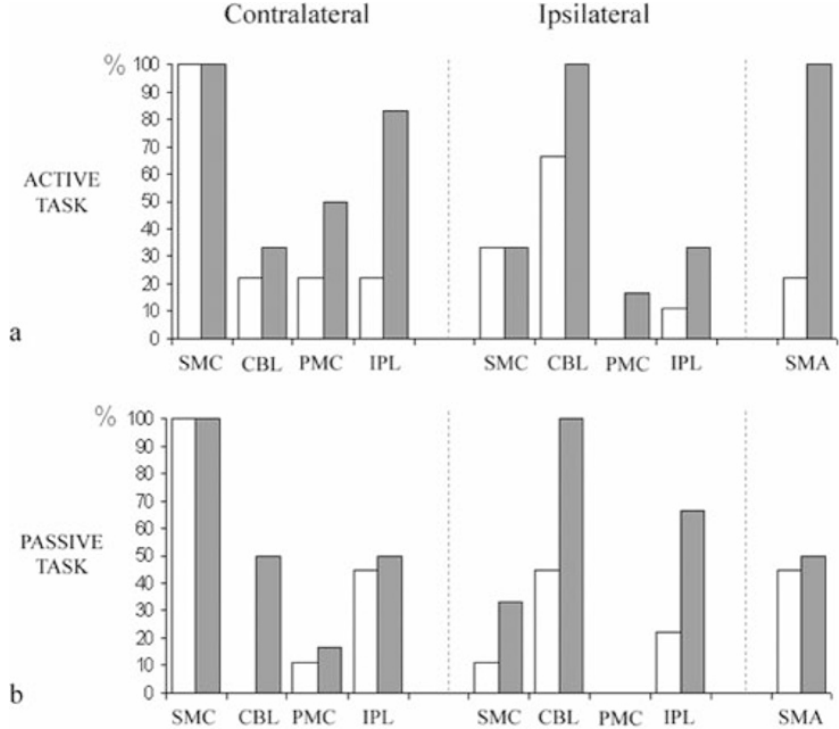

Figure 1. Percentage of subjects with significant activation for each brain region during active task $(a)$ and passive task $(b)$. CBL, cerebellum. Children (open columns), adults (shaded columns).

ondary somatosensory area, S2). The group analysis of the random-effect activation patterns did not show any area of significant difference between children and adults $(p>0.05$ corrected for multiple comparisons).

Brain representation of passive movements in children and adults. As for the active task, in each comparison of passive motor task and silent rest, for both the children and the adult groups, a large cluster of significantly activated voxels was always found in the contralateral primary SMC. Activated areas were also present in a variable proportion of subjects in the cerebellum, the SMA, the IPL, and the ipsilateral PMC (Fig. 1b). The pattern of brain activation was generally more widespread in the adult group, as shown by the presence of a higher proportion of active regions in adults compared with children. This pattern was confirmed by the number of activated voxels that was significantly different in the two groups, particularly in the contralateral SMC $(p<0.05)$ and in the ipsilateral cerebellum $(p<0.05)$. No significant difference was found for the SMA (Fig. 2b).

A visual comparison of the fixed-effect activation patterns after passive hand movement in the two groups revealed a high degree of similarity (Fig. 3). In both samples, the global maximum of activation was located in the contralateral rolandic region. Other large clusters of activation were detected at the level of the ipsilateral cerebellum and the contralateral IPL. A very small area of activation of the SMA was only present in children. The group analysis of the random-effect activation patterns did not show any area of significant difference between children and adults $(p>0.05$ corrected for multiple comparisons).

Comparison of active and passive movements. The group analysis of the random-effect activation patterns did not show any area of significant difference in activation between active and passive movement $(p>0.05)$.

The individual analysis of active $>$ passive motor task did not show any significant difference in seven of nine children 

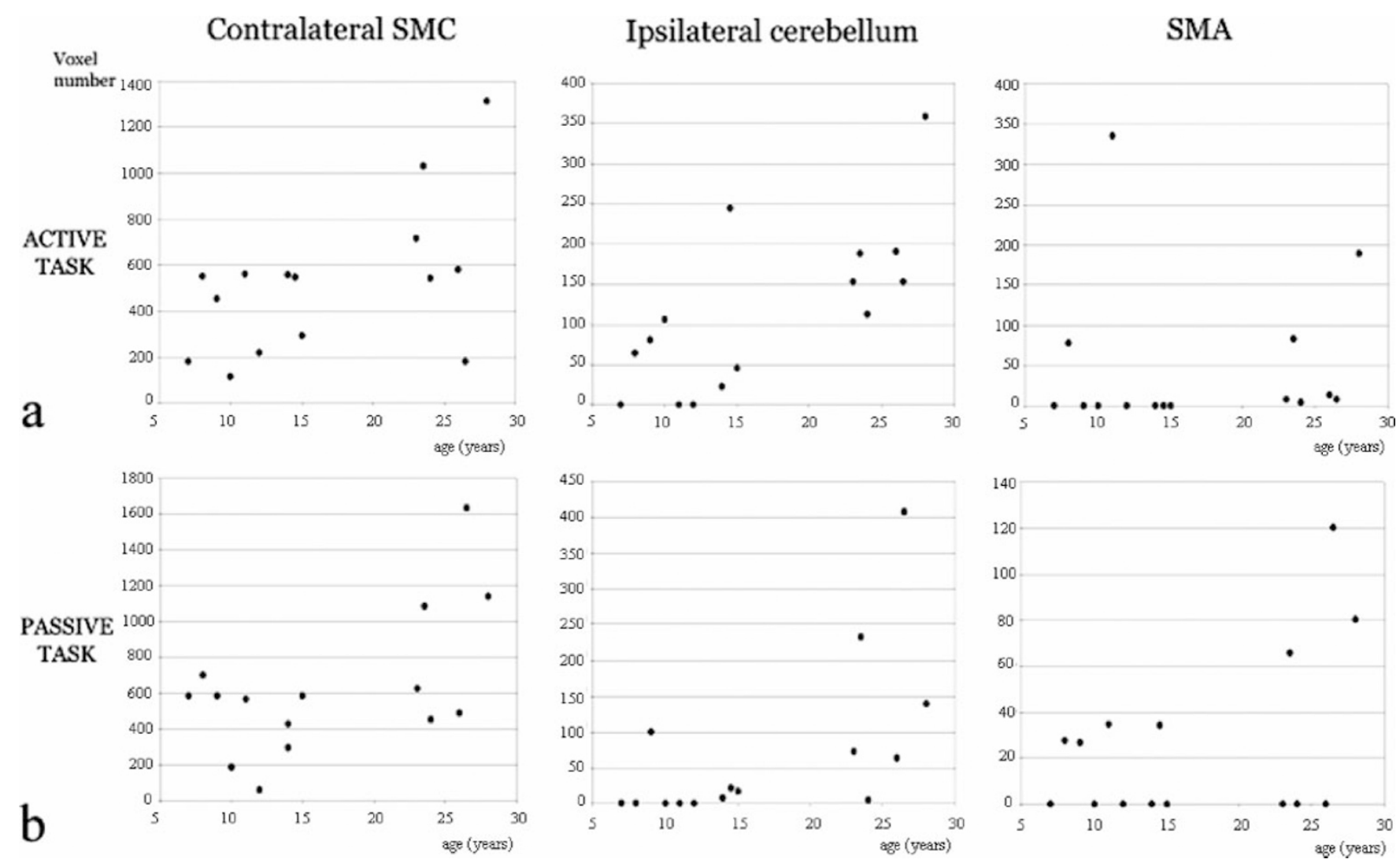

Figure 2. Active (a) and passive (b) hand movement $v s$ rest. Number of voxels activated (average of right and left hand movement) in different regions for each patient in relation to age (normalized data).

and in five of six adults. In all cases in which a difference was detected, it involved single small areas, always during right hand movement. The area involved was the contralateral rolandic region in one child, the SMA in the other and the ipsilateral ventral premotor region in the adult subject (Fig. 4). The individual analysis of passive $>$ active motor task did not show any significant difference in seven of nine children and in five of six adults. When a difference was detected, the activation involved the contralateral rolandic region in one child and one adult, and the bilateral rolandic regions in one child (Fig. 5).

Comparison of dominant and nondominant hands. To calculate the possible difference of activation produced by the movement of the dominant hand and the nondominant hand, we measured in each subject an asymmetry index based on the number of activated voxels in the various regions. The asymmetry index was calculated as follows: (dominant hand nondominant hand)/(dominant hand + nondominant hand). As a result, a positive index indicates greater activity after dominant hand tasks and a negative index indicates greater activity after nondominant hand tasks. Only the brain areas that were consistently activated in at least half of the subjects in the two groups were considered, i.e. the contralateral SMC and the ipsilateral cerebellum. The mean and SDs are shown in Figure 6. Comparison of the means in the two groups was obtained by using the two independent samples $t$ test, and no significant differences were detected.

\section{DISCUSSION}

In the past years, an increasing number of studies has been produced exploring the sensorimotor function in children with various neurologic disorders, in particular, in those with focal brain lesions and hemiplegia $(3,10,17,18)$. In these subjects, simple paradigms of hand opening and closing have proven particularly useful because they are easy to perform and need only a limited level of compliance. Nevertheless, brain representation of active and especially passive movements in children have been so far poorly investigated.

The active and passive movement tasks used in this study produced a consistent pattern of activation in the sensorimotor system of both children and adults. In particular, all subjects studied showed a robust activation of the contralateral SMC in both conditions. Variable proportions of subjects also activated a wider network of cortical areas including the ipsilateral SMC, the PMC, the SMA, the IPL, and cerebellum. This distribution was further confirmed by the fixed effect group analysis, which showed a consistent pattern of activation involving all the regions described. It is of interest that in all our children, and therefore from $7 \mathrm{y}$ onward, single-subject analysis was able to detect consistent activation of the primary SMC, with no exceptions, suggesting that this technique can be efficiently used for the exploration of the sensorimotor system even in childhood. 


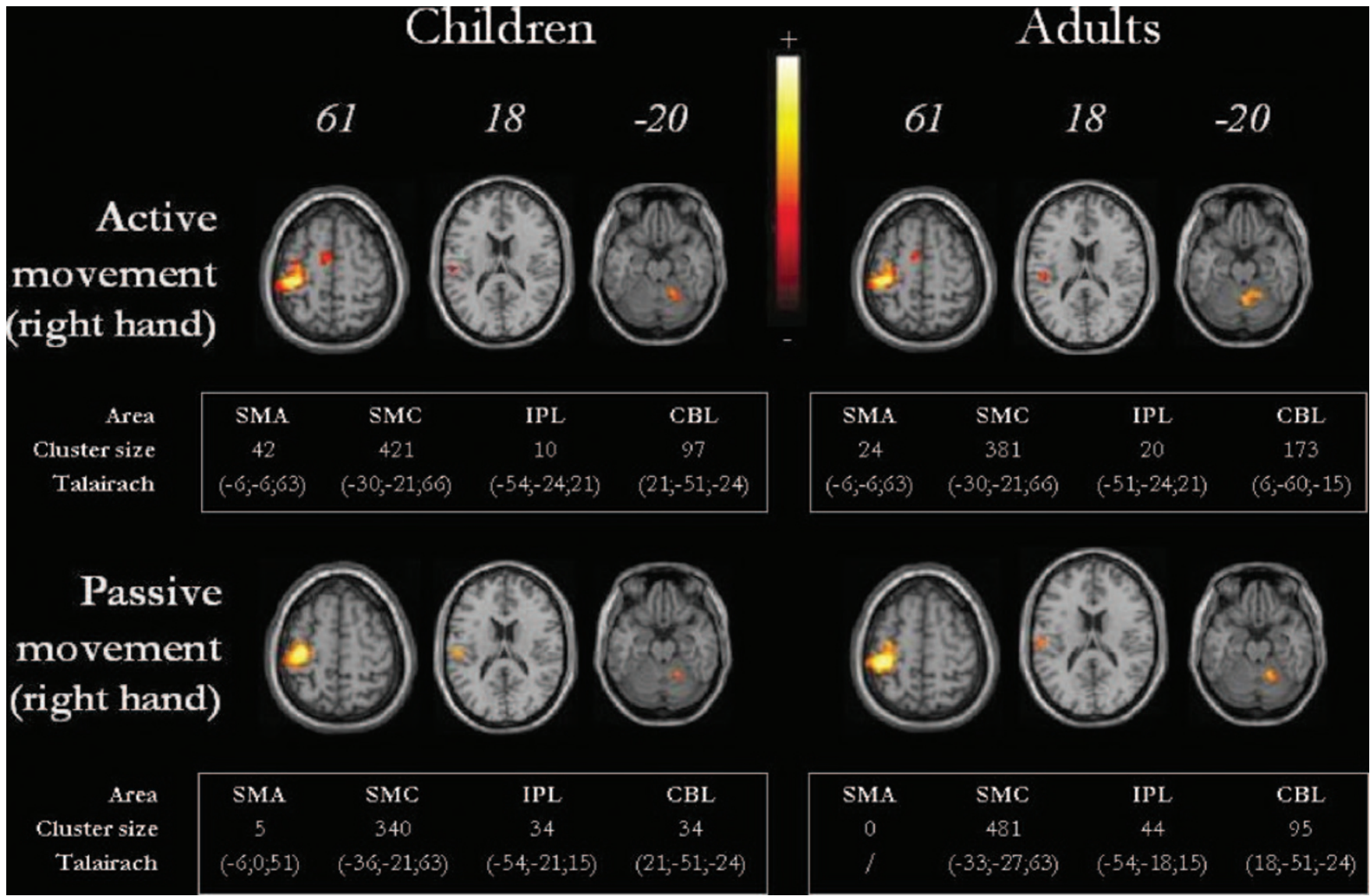

Figure 3. Fixed-effect analysis for children and adults during active and passive movements, using a $(p<0.05)$ activation threshold at the voxel level, corrected for multiple comparisons (FWE). Activation for each task is overlaid on a standard T1 brain image. Talairach $Z$ position of selected slices is indicated on the top. Cluster size and Talairach coordinates of activation maximum are shown for each area. CBL, cerebellum.

Apart from the primary SMC, for each of the other regions, the proportion of subjects with significant activation was always higher in the adult group. This finding was confirmed by quantitative analysis (voxel count), which showed significantly more widespread activation in the adult subjects in the contralateral SMC and in the ipsilateral cerebellum and a trend toward higher activations in the other regions. It has to be noted, however, that the more conservative group analysis based on random-effect activation patterns failed to show areas of significantly different activation when comparing children with adults and adults with children.
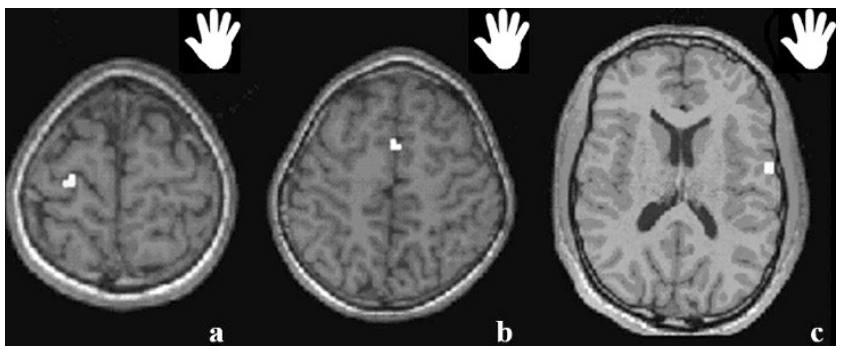

Figure 4. Active $>$ passive movement (right hand): subjects with areas of significantly different activation. (a) An 8-y-old child with a single area at the level of the left rolandic region. (b) An 11-y-old child with a single area of activation at the level of the SMA. (c) A 28-y-old adult subject with a single area of activation at the level of the right ventral premotor region.

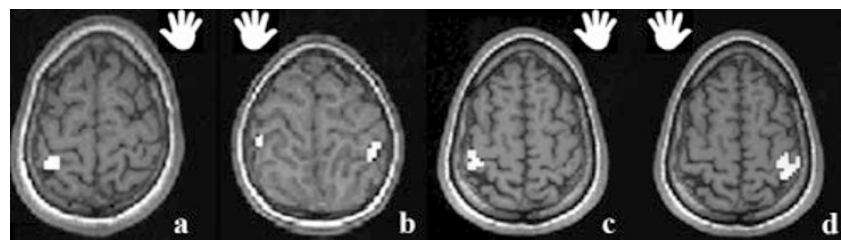

Figure 5. Passive $>$ active movement: subjects with areas of significant activation. (a) A 7-y-old child with a single area of activation at the level of the left rolandic region after right hand movement. (b) A 15-y-old child with bilateral small areas of activation at the level of the rolandic regions after left hand movement. (c) A 26-y-old adult subject with a single area of activation at the level of the left rolandic region after right hand movement. (d) Same subject as in $c$ with a single area of activation at the level of the right rolandic region after left hand movement.

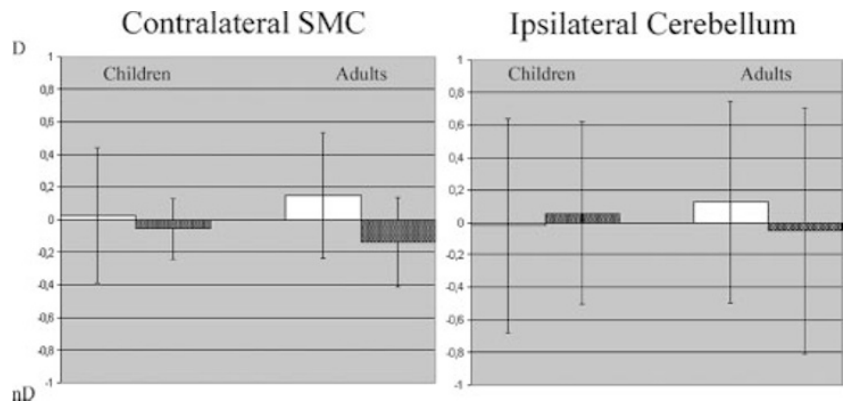

Figure 6. Asymmetry indexes in children and adults. See text for details. D, dominant hand; $\mathrm{nD}$; nondominant hand. Active task (open bars); passive task (shaded bars). 
The presence of a different pattern of activation during development, as opposed to adult age, has been recently reported in relation to active movement by Mall et al. (13), who showed a significant difference in the degree of cortical activation of the bilateral SMC, parietal areas, SMA, and cerebellum. Our results are partly in accordance with those of this study, which is the only one comparing the motor function in children and adults by means of fMRI; nevertheless, the two studies are not easy to compare as several relevant methodological differences are present. First, our groups were different in size and age range. In particular, we considered in the children group all subjects between 7 and $15 \mathrm{y}$ of age, whereas in the study by Mall et al. this same age range defined two different groups, children from 6 to $10 \mathrm{y}$ and adolescents from 11 to $15 \mathrm{y}$. Second, we did not use a squeeze ball in our task because we were interested in a simple task that was comparable (i) with that used in previous studies performed in children with hemiplegia and (ii) with the matching task of passive movement, which clearly could not be performed with the use of this tool.

This is the first study investigating the developmental aspects of passive movement representation in children. As for the active task, we have shown significantly stronger activation of the main areas involved in passive movement, i.e. the contralateral SMC, ipsilateral cerebellum, and SMA. This difference was again not strong enough to reach significance after more conservative analyses. The distribution of the activation in children was also consistent with that of previous reports on passive movements in adult subjects with different types of tasks. Weiller et al. (9) were the first to explore the representation of passive movements in humans by means of positron emission tomography (PET) in an elbow flexion/ extension experiment. They showed that passive movement was able to produce activation of most of the cortical areas involved in motor control, such as the contralateral primary SMC, the SMA, and the IPL. This result was eventually confirmed by other authors using different types of passive tasks $(19,20)$. Less consistent in the literature are the results concerning ipsilateral cerebellum activation during passive tasks. Several authors reported an absence of cerebellar activation after tactile/vibratory, nociceptive, and proprioceptive stimulation in adults $(9,21-25)$. Conversely, an essential contribution of the cerebellum in somatosensory perception has been recently suggested by studies on tactile discrimination and passive limb movements, both performed and imagined (20,26-30). Accordingly, it is of interest how, in our sample, activation of the ipsilateral cerebellum after passive hand movement was present in all the adult subjects and, with smaller number of activated voxels, in almost half of our children.

It is important to consider that the differences detected between children and adults in terms of cortical activation may be related to an age dependency of the blood oxygen level-dependent (BOLD) signal. Developmental influences on the BOLD signal have been recently investigated in a large cohort of children by Schapiro et al. (31) during sensorimotor and language tasks. An increase in the BOLD effect during childhood occurring selectively in a subset of task-related regions was consistently shown, suggesting that brain activation as measured by fMRI may be used as a marker of regional brain specialization during development. Our results are in full agreement with this hypothesis, but other confounding factors such as a difference in the quality of the performance cannot be excluded. To limit the presence of this bias, we used an active motor task not requiring any extra amount of muscle power other than that needed for joint displacement, whereas for the passive task, the same examiner performed the passive movement in all subjects. Despite that, subtle differences related to segment size, muscle power, and degree of joint displacement cannot be fully overcome.

It has to be emphasized that the purpose of our study was not to establish a normative reference for active and passive hand movement representation in children. This would be hardly feasible even with large cohorts, due not only to the difficulty of task performance standardization, but especially to the methodological limits of group comparisons of different age groups because the templates used (either based on normal adult or child populations) determine the degrees of warping errors largely not comparable. Accordingly, for the purposes of this study, we mainly based our analysis on general visual observation of individual cases.

Brain structures activated by active and passive movements in our study were consistently overlapping. When contrasting active versus passive tasks, no difference was detected in seven of nine children and in five of six adults, whereas when a difference was found, it was always small and limited to one or two areas. These minor differences were no longer evident after group analysis and are therefore more likely related to different levels of regional activation and not to topographic distinctions. Our findings are in agreement with those of previous adult studies comparing an active motor task with passive movement (9) and with sensory stimulation (32-34), in which a tight coupling between sensory and motor cortical activation was found. Different results were obtained by Mima et al. (35), who studied finger active and passive movement with PET, showing a weaker and spatially more circumscribed activation after passive movement. A large part of the differences may be related to the different methodologies that were used. In particular, in contrast to our study, these authors devised a passive task selectively activating proprioception with a minimal contribution from the tactile senses. It has been proposed that such a task can produce very limited somatosensory attention, i.e. the discrimination or categorization of the somatosensory stimulation, which has been shown to be able itself to activate neurons of the SMC and the SMA $(36,37)$. This might explain the different results reached by these authors in that larger and less selective passive movements may be responsible for an implicit involvement of the somatosensory attentional shift.

When analyzing the correlation between handedness and the extension of the activation in the contralateral SMC and the ipsilateral cerebellum, we did not see any statistically significant difference in children or in adults. This finding is not surprising because there is no agreement in the literature on the handedness-related asymmetries of brain activation, despite the good evidence of anatomical asymmetries, espe- 
cially regarding the PMC, consisting of more extensive representation of regions contralateral to the dominant hand (38). A possible interpretation is that the area activated in each hemisphere during movement of the contralateral hand reflects the functional demands of task performance of each hand rather than the underlying anatomical limits (39). In this view, a handedness-related differential activation, with larger areas of activation in the nondominant hemisphere, would be progressively more obvious with increased difficulty of the motor task, but poorly evident after simpler tasks, as the ones used in our study.

In conclusion, our results suggest that active and passive hand movements can be effectively used for the exploration of the sensorimotor system in children, thus sustaining previous findings on functional reorganization of children with congenital brain lesions. Good compliance and reliable results can be easily obtained in children, even with very simple motor tasks. Moreover, we have confirmed in a young population the tight coupling between the active and passive tasks already shown in adult subjects, thus supporting the idea of a possible use of the passive task as a helpful performance-independent paradigm in the study of brain reorganization in children with brain lesions as well as in preoperative evaluation of young children who are candidates for neurosurgery.

\section{REFERENCES}

1. Frackowiak RS, Ashburner JT, Penny WD, Zeki S, Friston KJ, Frith CD, Dolan RJ, Price CJ 2004 Human brain function, $2^{\text {nd }}$ edition. Academic Press, New York

2. Colebatch JG, Deiber MP, Passingham RE, Friston KJ, Frackowiak RS 1991 Regional cerebral blood flow during voluntary arm and hand movements in human subjects. J Neurophysiol 65:1392-1401

3. Holloway V, Gadian DG, Vargha-Khadem F, Porter DA, Boyd SG, Connelly A 2000 The reorganization of sensorimotor function in children after hemispherectomy. A functional MRI and somatosensory evoked potential study. Brain 123:2432-2444

4. Hutchinson S, Kobayashi M, Horkan CM, Pascual-Leone A, Alexander MP, Schlaug G 2002 Age-related differences in movement representation. Neuroimage 17:17201728

5. Jenkins IH, Jahanshahi M, Jueptner M, Passingham RE, Brooks DJ 2000 Selfinitiated versus externally triggered movements. II. The effect of movement predictability on regional cerebral blood flow. Brain 123:1216-1228

6. Nelles G, Spiekermann G, Jueptner M, Leonhardt G, Muller S, Gerhard H, Diener HC 1999 Reorganization of sensory and motor systems in hemiplegic stroke patients. A positron emission tomography study. Stroke 30:1510-1516

7. Remy P, Zilbovicius M, Leroy-Willig A, Syrota A, Samson Y 1994 Movement- and task-related activations of motor cortical areas: a positron emission tomographic study. Ann Neurol 36:19-26

8. Roland PE, Larsen B, Lassen NA, Skinhoj E 1980 Supplementary motor area and other cortical areas in organization of voluntary movements in man. J Neurophysiol 43:118-136

9. Weiller C, Juptner M, Fellows S, Rijntjes M, Leonhardt G, Kiebel S, Muller S, Diener HC, Thilmann AF 1996 Brain representation of active and passive movements. Neuroimage 4:105-110

10. Vandermeeren Y, Sebire G, Grandin CB, Thonnard JL, Schlogel X, De Volder AG 2003 Functional reorganization of brain in children affected with congenital hemiplegia: fMRI study. Neuroimage 20:289-301

11. Cao Y, D’Olhaberriague L, Vikingstad EM, Levine SR, Welch KM 1998 Pilot study of functional MRI to assess cerebral activation of motor function after poststroke hemiparesis. Stroke 29:112-122

12. Marshall RS, Perera GM, Lazar RM, Krakauer JW, Constantine RC, DeLaPaz RL 2000 Evolution of cortical activation during recovery from corticospinal tract infarction. Stroke 31:656-661
13. Mall V, Linder M, Herpers M, Schelle A, Mendez-Mendez J, Korinthenberg R, Schumacher M, Spreer J 2005 Recruitment of the sensorimotor cortex-a developmental FMRI study. Neuropediatrics 36:373-379

14. Klose U, Erb M, Wildgruber D, Muller E, Grodd W 1999 Improvement of the acquisition of a large amount of MR images on a conventional whole body system. Magn Reson Imaging 17:471-474

15. Friston KJ, Holmes AP, Worsley KJ, Poline JB, Frith CD, Frackowiak RS 1995 Statistical parametric maps in functional imaging: a general approach. Hum Brain Mapp 2:189-210

16. Friston KJ, Holmes AP, Price CJ, Buchel C, Worsley KJ 1999 Multisubject fMRI studies and conjunction analyses. Neuroimage 10:385-396

17. Staudt M, Gerloff C, Grodd W, Holthausen H, Niemann G, Krägeloh-Mann I 2004 Reorganization in congenital hemiparesis acquired at different gestational ages. Ann Neurol 56:854-863

18. Staudt M, Grodd W, Gerloff C, Erb M, Stitz J, Krägeloh-Mann I 2002 Two types of ipsilateral reorganization in congenital hemiparesis: a TMS and fMRI study. Brain $125: 2222-2237$

19. Alary F, Doyon B, Loubinoux I, Carel C, Boulanouar K, Ranjeva JP, Celsis P, Chollet F 1998 Event-related potentials elicited by passive movements in humans: characterization, source analysis, and comparison to fMRI. Neuroimage 8:377-390

20. Carel C, Loubinoux I, Boulanouar K, Manelfe C, Rascol O, Celsis P, Chollet F 2000 Neural substrate for the effects of passive training on sensorimotor cortical representation: a study with functional magnetic resonance imaging in healthy subjects. J Cereb Blood Flow Metab 20:478-484

21. Burton H, Videen TO, Raichle ME 1993 Tactile-vibration-activated foci in insular and parietal-opercular cortex studied with positron emission tomography: mapping the second somatosensory area in humans. Somatosens Mot Res 10:297-308

22. Fox PT, Burton H, Raichle ME 1987 Mapping human somatosensory cortex with positron emission tomography. J Neurosurg 67:34-43

23. Seitz RJ, Roland PE 1992 Vibratory stimulation increases and decreases the regional cerebral blood flow and oxidative metabolism: a positron emission tomography (PET) study. Acta Neurol Scand 86:60-67

24. Tempel LW, Perlmutter JS 1992 Vibration-induced regional cerebral blood flow responses in normal aging. J Cereb Blood Flow Metab 12:554-561

25. Casey KL, Minoshima S, Morrow TJ, Koeppe RA 1996 Comparison of human cerebral activation pattern during cutaneous warmth, heat pain, and deep cold pain. J Neurophysiol 76:571-581

26. Naito E, Kochiyama T, Kitada R, Nakamura S, Matsumura M, Yonekura Y, Sadato N 2002 Internally simulated movement sensations during motor imagery activate cortical motor areas and the cerebellum. J Neurosci 22:3683-3691

27. Jueptner M, Ottinger S, Fellows SJ, Adamschewski J, Flerich L, Muller SP, Diener HC, Thilmann AF, Weiller C 1997 The relevance of sensory input for the cerebellar control of movements. Neuroimage 5:41-48

28. Gao JH, Parsons LM, Bower JM, Xiong J, Li J, Fox PT 1996 Cerebellum implicated in sensory acquisition and discrimination rather than motor control. Science 272:545-547

29. Liu Y, Pu Y, Gao JH, Parsons LM, Xiong J, Liotti M, Bower JM, Fo PT 2000 The human red nucleus and lateral cerebellum in supporting roles for sensory information processing. Hum Brain Mapp 10:147-159

30. Parsons LM, Bower JM, Gao JH, Xiong J, Li J, Fox PT 1997 Lateral cerebellar hemispheres actively support sensory acquisition and discrimination rather than motor control. Learn Mem 4:49-62

31. Schapiro MB, Schmithorst VJ, Wilke M, Byars AW, Strawsburg RH, Holland SK 2004 BOLD fMRI signal increases with age in selected brain regions in children. Neuroreport 15:2575-2578

32. Puce A, Constable RT, Luby ML, McCarthy G, Nobre AC, Spencer DD, Gore JC, Allison T 1995 Functional magnetic resonance imaging of sensory and motor cortex: comparison with electrophysiological localization. J Neurosurg 83:262-270

33. Yetkin FZ, Mueller WM, Hammeke TA, Morris GL3rd, Haughton VM 1995 Functional magnetic resonance imaging mapping of the sensorimotor cortex with tactile stimulation. Neurosurgery 36:921-925

34. Lee CC, Jack CR Jr, Riederer SJ 1998 Mapping of the central sulcus with functional MR: active versus passive activation tasks. AJNR Am J Neuroradiol 19:847-852

35. Mima T, Sadato N, Yazawa S, Hanakawa T, Fukuyama H, Yonekura Y, Shibasaki H 1999 Brain structures related to active and passive finger movements in man. Brain 122:1989-1997

36. Mountcastle VB, Atluri PP, Romo R 1992 Selective output-discriminative signals in the motor cortex of waking monkeys. Cereb Cortex 2:277-294

37. Romo R, Ruiz S, Crespo P, Zainos A, Merchant H 1993 Representation of tactile signals in primate supplementary motor area. J Neurophysiol 70:2690-2694

38. Hammond G 2002 Correlates of human handedness in primary motor cortex: a review and hypothesis. Neurosci Biobehav Rev 26:285-292

39. Volkmann J, Schnitzler A, Witte OW, Freund H 1998 Handedness and asymmetry of hand representation in human motor cortex. J Neurophysiol 79:2149-2154 